\title{
Complex method of peen forming and shot peening of aircraft structural components
}

\author{
Pashkov Andrey E. \\ Institute of aircraft and machine building and transport \\ (IAMBT) \\ Irkutsk National Research Technical University \\ Irkutsk, Russia \\ pashkov@istu.edu
}

\author{
Pashkov Aleksander A. \\ Institute of aircraft and machine building and transport \\ (IAMBT) \\ Irkutsk National Research Technical University \\ Irkutsk, Russia
}

\author{
Koltsov Vladimir P. \\ Institute of aircraft and machine building and transport (IAMBT) \\ Irkutsk National Research Technical University \\ Irkutsk, Russia
}

\begin{abstract}
The paper describes a complex method for forming aircraft panels as well as automated plants for bending, peen forming, sanding, and shot peening. It also describes calculation methods for technological conditions which compensate for the effect of the residual stress on the shape of parts.
\end{abstract}

Keywords - panel, skin, bending, peenforming, sanding, shot peening, residual stress.

\section{INTRODUCTION}

Forming large-size sheet skins and stiffened aircraft panels is a major technological concern due to the following factors. First, aircraft surfaces have small curvatures which can be obtained by overall strains comparable to elastic components. Second, large dimensions of panels and internal stiffeners make it difficult to employ forming methods for the whole surface of the part by means of fixtures fitted for its shape. In this regard, methods of local strains (free bending and peen forming) became common in producing aircraft panels and skins.

Peen forming involves biaxial stretching of surface layers by shots. The technique allows forming surfaces of sheet panels with double curvatures. To obtain surfaces of the prescribed shape for wing skins, prestressing increasing the curvature in the direction of the preliminary elastic bend, as well as two-sided peen forming of the outer surface of panels and their edges, are used. Forming a bend - an area of the alternating double curvature - is especially difficult. It is a multistage process combining shot peening of various diameters, prestressing and edge stretching [1].

That technique was patented by Boeing Company and involves peen forming operations: two-sided peen forming of the panel to obtain the longitudinal curvature, external peen forming of the panel to obtain the transversal curvature, surface sanding, and shot peening. Forming conditions are determined based on databases, which contain dependences of two-sided sample elongations and transversal curvature of onesided samples on the Almen intensity [2]. Two shot blasters are used for panel forming. The first one is used to obtain the longitudinal curvature by two-sided peen-forming of edges of 3.2. $\mathrm{mm}$ in diameter. It is equipped with four movable shot blasters (two on each side). The second one is used to treat the outer surface of the panel by the shot of $1.12 \mathrm{~mm}$ in diameter. For this purpose, 12 fixed shot blasters (six on each side for forming of left and right parts of the wing) are used [3].

The peculiarity of the method is due to the fact that peen forming is carried out in closed chambers, and the final part is shaped upon the completion of the process. So, large volume of experimental work is required for developing monitoring programs. Such expenses do not ensure required accuracy. As mentioned in [2], the accuracy of a part profile is $1.3 \mathrm{~m}$ with permissible load of $45.36 \mathrm{~kg}$ on the section. It means that the use of expensive and technically complex equipment solves the issue of operation automation only for preliminary (rough) forming. To obtain a profile of the required accuracy, handcontrolled equipment and checking fixture are needed.

\section{METHOD DESCRIPTION}

The Russian alternative to the foreign technologies is a complex method for skin and panel forming developed by Irkutsk National Research Technical University for Irkutsk Aviation Plant - Branch of JSC "Irkut" Corporation. The main technological advantage of the complex forming method (Fig. 1 ) is the process subdivision into simple, easily operated and controlled stages for obtaining longitudinal and transversal curvatures of parts. 

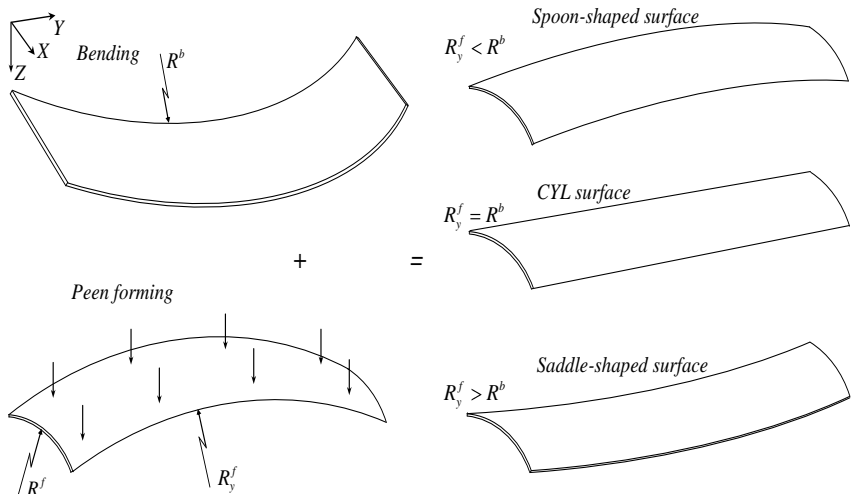

Fig. 1. Scheme of panel forming

The longitudinal curvature of sheet panels (e.g., skins) can be obtained by bending on rollers. For monolithic ribbed panels, rib rolling is used. The transversal curvature can be obtained by peen forming of the outer surface of a part by balls of 3-4 $\mathrm{mm}$ in diameter, followed by sanding for the rough surface. Peen forming and sanding are followed by two-sided shot peening by shots of 0.6-0.8 $\mathrm{mm}$ in diameter.

A special automated plant is used for forming parts.

Bending is performed on a sheet-bender (Fig. 2) modernized by replacing drive mechanisms of rolls and implementing a program control system.

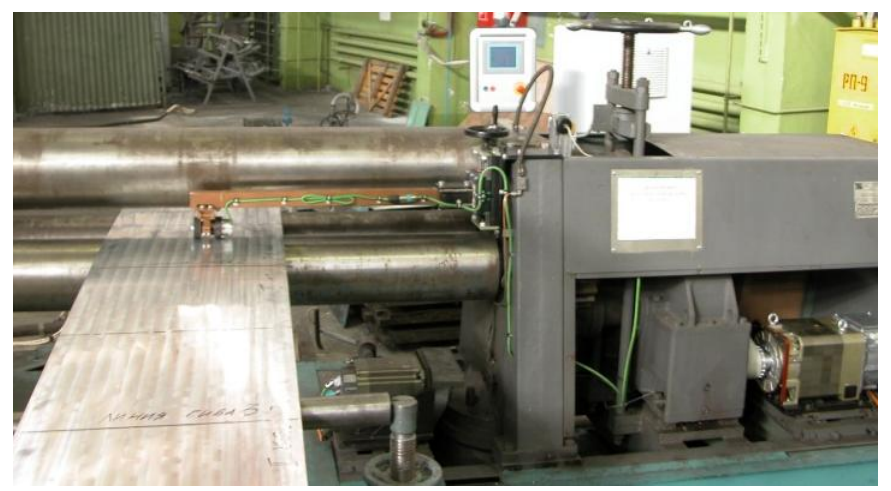

Fig. 2. Sheet-bender I2222BM

The rolling plant (Fig. 3) was developed for transversal bending of stiffened monolithic ribbed panels (Fig. 3).

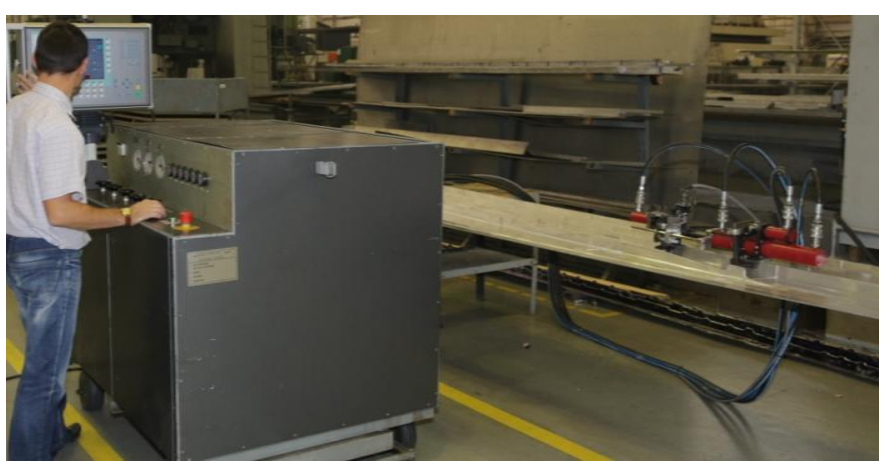

The research was carried out with funding from the Ministry of Education and Science of the Russian Federation

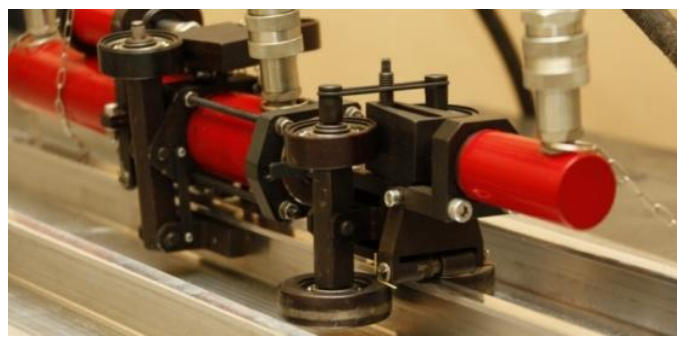

Fig. 3. Roller for large-size ribbed panels

In contrast to foreign equipment, UDF-4, applied for peen forming and sanding, uses contact-type operating elements moving along a fixed part (Fig. 4).

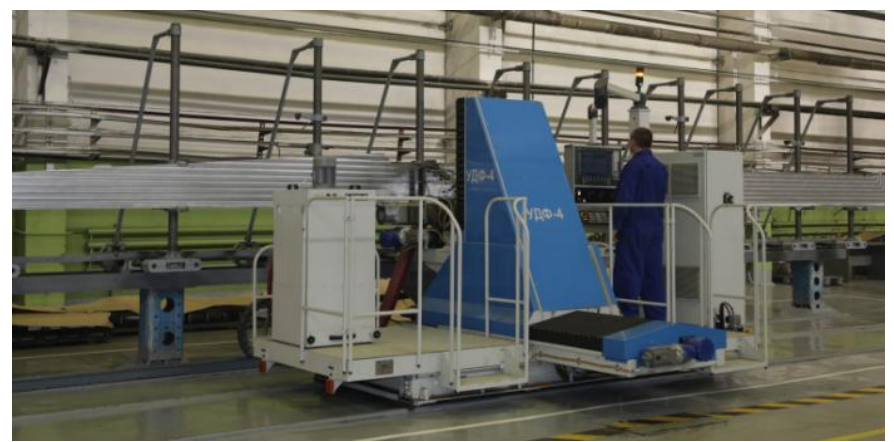

Fig. 4. Peen-forming and sanding plant UDF-4

UDF-4 is equipped with two changeable operating elements - a shot-blaster (Fig. 5, a) and a sanding head (Fig. 5, b).

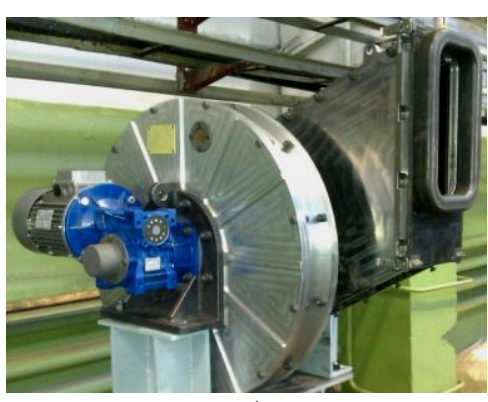

a)

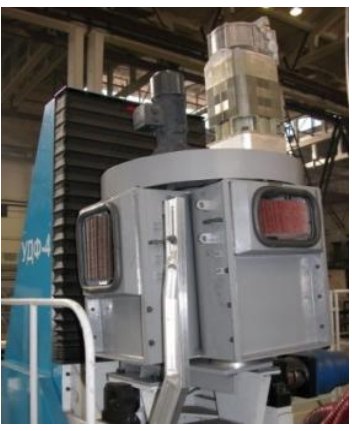

b)
Fig. 5. Operating elements of UDF-4: a) a shot-blaster; b) a sanding head

UDP-2,2.5 Shot-blaster is used for shot peening of long parts up to $2.5 \mathrm{~m}$ height (Fig. 6). 


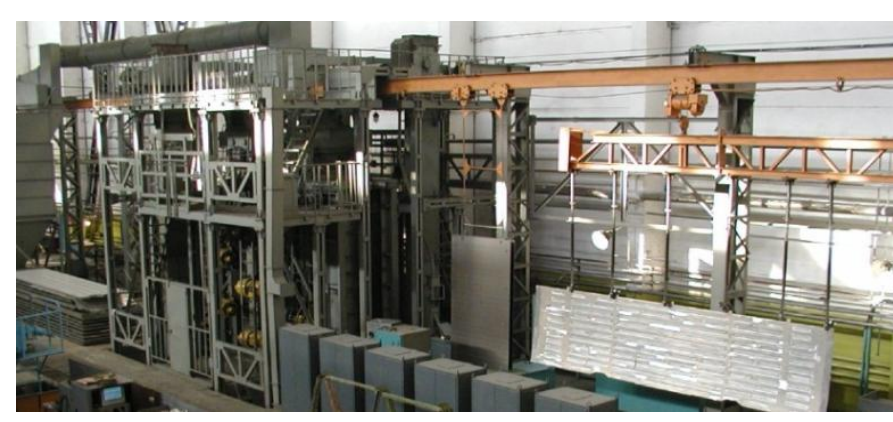

Fig. 6. Automated shotblaster UDP-2-2.5

\section{STRESS-STRAIN STATE OF PARTS FORMED BY PEEN FORMING}

For mathematical description of stress-strain state of the part formed by peen forming, we use an approach based on the concept of inherent stresses - elastic stresses unbalanced in the section of the part resulting from plastic strains, phase transformations, etc. [4]. When balancing inherent stresses, the internal force and bending moment occur which cause distortion of thin-walled parts.

When arranging origins of coordinates on the surface treated, the bending moment resulting from the technological impact is determined as (1):

$$
M_{j}=P_{j}\left(z_{0 k}-z_{c k}\right), j, k=x, y ;
$$

where $P$ is the internal force due to peen forming, being an integral value of distribution of inherent stresses; $z_{c}$ is the coordinate of the point of force application; $z_{0}$ is the coordinate of a neutral layer of the section. Therefore, to determine changes in part shape, resulting from surface forming, one needs to calculate internal force factors $P$ and $z_{c}$ which determine the strain value for a part at all stages of the process. $z_{c}$

Fig. 7 shows formalized distributions of inherent stresses when forming a part.

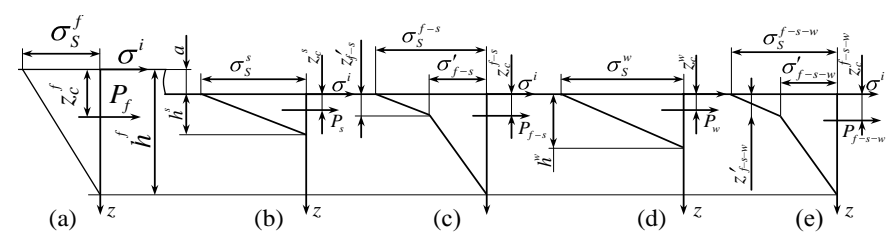

Fig. 7 Distributions of inherent stresses caused by peen forming (a) cutting during sanding (b); sequence 'peen forming - sanding' (c); shot peening (d); sequence 'peen forming - sanding - shot peening (e)

The resultant distributions shown in Fig. 7c and 7e can be obtained if one take into account the characteristics of stress state formation under multiple loading. According to [5], during subsequent loading coincidental with the previous one by direction, elastic uploading is followed by plastic strains which occur in the point corresponding to the value of von Mises flow stress of the previous loading. Assuming the similarity of stress state of processes under study, the higher value of inherent stresses is considered resultant.
For analytical description of distributions of inherent stresses, the authors use linear approximations. Researchers of shot-peen forming processes [6] use the triangular approximation in inherent and residual stresses in the surface layer (see (2)):

$$
\sigma^{i}=\sigma_{S}(1-z / h)
$$

To analyze resultant distributions of inherent stresses (see Fig. 7c and 7e), the authors use a polygonal function consisting of two parts (see (3)):

$$
\begin{aligned}
\sigma^{i} & =\sigma_{S}+\frac{z}{z^{\prime}}\left(\sigma^{\prime}-\sigma_{S}\right) ; z<z^{\prime} ; \\
\sigma^{i} & =\sigma^{\prime}\left(1-\frac{z-z^{\prime}}{h-z^{\prime}}\right) ; z^{\prime}<z<h .
\end{aligned}
$$

Here, $z^{\prime}$ is coordinate of the intersection point for the linear approximations.

Based on (3), one can obtain (4) for calculating internal force factors $P$ and $z_{c}$. For example, for the polygonal approximation:

$$
P=-\frac{1}{2} \sigma_{S} z^{\prime}+\sigma^{\prime} h ; z_{c}=\frac{\sigma_{S} z^{2}+\sigma^{\prime}\left(3 z^{\prime 2}-2 z^{\prime} h+h^{2}\right)}{3\left(\sigma_{S} z^{\prime}+\sigma^{\prime} h\right)}
$$

After transformation of the equation for calculating resultant force $P_{f-s-w}$, acting on the outer side of the part and coordinates of the point of its application, $z_{c}^{f-s-w}$ can be written in the form (5):

$$
P_{f-s-w}=-\frac{1}{2}\left[\sigma_{S}^{w} z_{f-s-w}^{\prime}+\sigma_{f-s-w}^{\prime}\left(h_{f}-a\right)\right] ;
$$

$z_{c}^{f-s-w}=\frac{\sigma_{S}^{w} z_{f-s-w}^{\prime 2}+\sigma_{f-s-w}^{\prime}\left[3 z_{f-s-w}^{\prime 2}-2 z_{f-s-w}^{\prime}\left(h_{f}-a\right)+\left(h_{f}-a\right)^{2}\right]}{3\left[\sigma_{S}^{w} z_{f-s-w}^{\prime}+\sigma_{f-s-w}^{\prime}\left(h_{f}-a\right)\right]}$.

The dependences determine force factors resulting in part forming based on the known parameters of distributions of inherent stresses caused by peen forming, sanding and shot peening.

When calculating integral parameters of distributions of inherent stresses caused by surface treatment, the authors use the methods suggested in [4]. They are based on measurements of strain bending and elongation of sample strips treated under the conditions under study.

To transfer from sample strips to plate-type parts, let us assume that

- under similar conditions, plastic strain distribution depths for samples and parts are equal;

- inherent stresses are distributed according to the linear law described by formula (2). 
The equations for calculating parameters of the triangular distribution of inherent stresses depending on the measured strain of the samples are as follows (6):

$$
h=\frac{3}{2} H-2 \frac{f}{\varepsilon}\left(\frac{H}{L}\right)^{2} ; \sigma_{S}=\frac{3 E H \varepsilon}{h} .
$$

The values of surface inherent stresses $\sigma_{S}$ and depths of their distribution $h$ for peen forming, sanding and shot peening calculated by means of equation (6) allow one to calculate the parameters of approximation of the process combination diagrams and calculate internal forces using (2) and (3). Integral parameters of distributions of inherent stresses created by peen forming, sanding and shot peening are calculated for the whole range of technological conditions and stored in databases.

Based on the non-linear finite element analysis, one can calculate distribution parameters for inherent stresses produced by peen forming by implementing a ball indentor with a diameter equal to the diameter of the shot. Figure 8 shows MSC Marc-based simulation results for the single implementation of a shot.

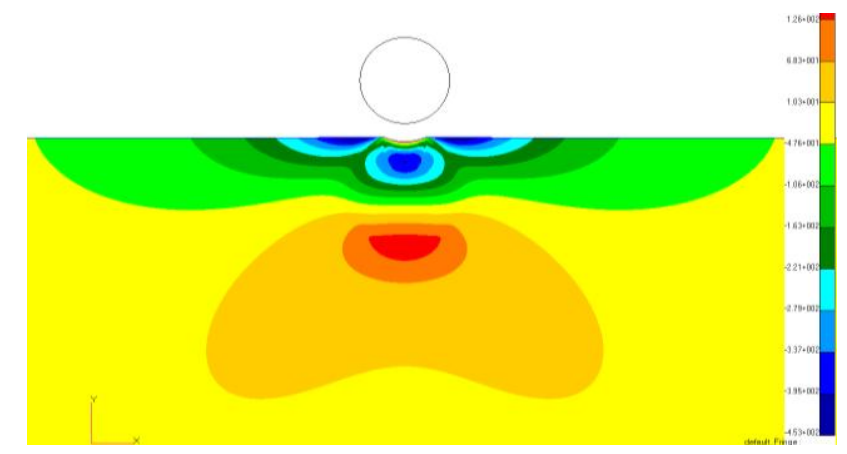

Fig. 8. Distribution of residual stresses after implementing a ball with a $3 \mathrm{~mm}$ diameter into a half-space with material properties of the parts treated.

Figure 9 shows the scheme of the residual stress-strain state of the single implementation based on simulation results.

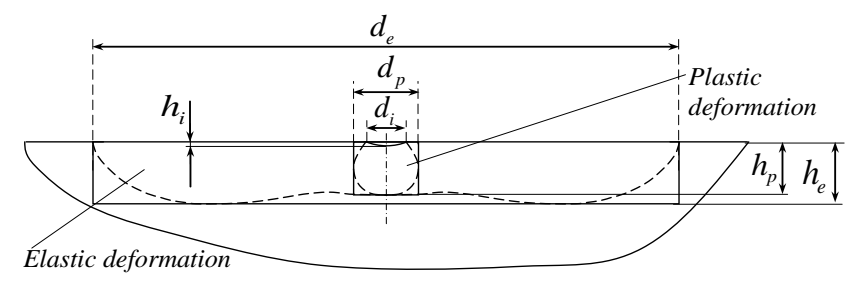

Fig. 9. Scheme of the residual stress-strain state of the single implementation

Figure 10 shows an averaged distribution of residual stresses for implementing a shot at different velocities.

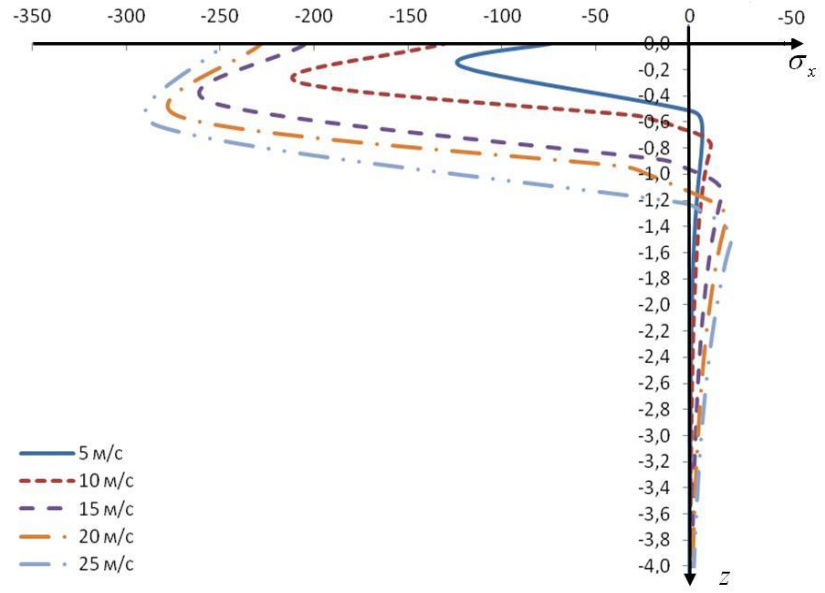

Fig. 10. Averaged residual stress distributions

Contact type shot-blasters have indentations of different diameters in the area treated (Fig. 11).

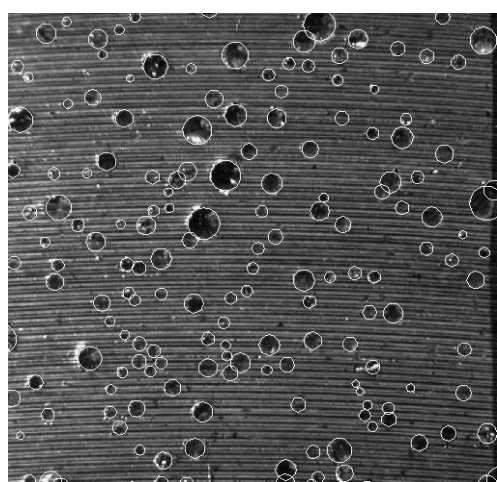

Fig. 11. Structure of the treated area for a contact type shot-blaster

To determine the averaged residual stresses in surface layers of the part, one can apply (7):

$$
\sigma_{r}=\sum_{k=1}^{N_{f}}\left(\sigma_{r p_{k}} S_{p_{k}}+\sigma_{e p_{k}} S_{e k}\right)
$$

where $N_{f}$ is the number of indentation fractions in treated area; $\sigma_{r_{p_{k}}}, \sigma_{e_{p_{k}}}$ are residual stresses in plastic deformation zones and elastically deformed zones if single implementation occurs; $S_{p_{k}}, S_{e k}$ are the degrees of coverage by indentations of k- fraction ( $S=F_{i n} / F, F$ is the total area of the treated part; $F_{i n}$ is the total area of deformation zones).

Given that the diameters of all deformation zones are equal, one can determine the degree of coverage by rewriting the formula in [6] as:

$$
S=1-\left[1-\pi d^{2} / 4 F_{e}\right]^{N},
$$

where $d$ is the diameter of a single indentation; $F_{e}$ is the area of a single surface treated; $N$ is the number of indentations.

If there are indentations of different diameters in the area treated, the degree of coverage can be determined by overlapping the second and subsequent indentions. Let us 
assume that a single area is sequentially treated with a stream of shots having equal velocities. During treatment of a single area with k-portion of shots, the degree of coverage by indentations of $\mathrm{k}$-fractions is:

$$
S_{k}^{\prime}=1-\left[1-\pi d_{k}^{2} / 4 F_{e}\right]^{N_{k}}
$$

where $d_{k}$ is the diameter of indentations of k-fraction; $N_{k}$ is the number of indentations of $\mathrm{k}$-fraction formed in a time unit.

For sequential process, the degree of coverage of each subsequent layer of indentations decreases due to the probability of their coincidence with the previous indentations, i.e.:

$$
S_{1}=S_{1}^{\prime} ; S_{2}=S_{2}^{\prime}\left(1-S_{1}\right) ; S_{3}=S_{3}^{\prime}\left(1-S_{1}-S_{2}\right)
$$

etc. As a result, the equation of a degree of coverage by indentations of $\mathrm{k}$-fraction for the recovering is:

$$
S_{k}=S_{k}^{\prime}\left(1-\sum_{i=1}^{k-1} S_{i}\right) \text {. }
$$

The resultant degree of coverage constitutes a sum:

$$
S=\sum_{k=1}^{N_{k}} S_{k}
$$

Thus, (7) allows one to calculate averaged residual stresses based on the structure of the area treated.

To determine the inherent stresses used for calculation of internal force factors, based on (2)-(4), let us present residual stress formation as a scheme (Fig. 12).

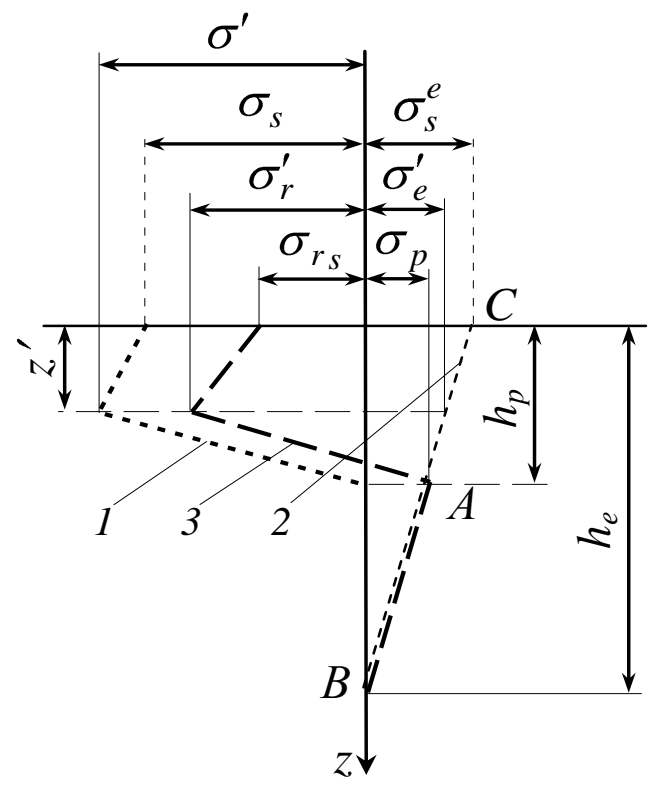

Fig. 12. Residual stress formation produced by shot peening: 1 - inheren stresses; 2 - reactive elastic stresses; 3 - residual stresses (sum of 1 and 2)

Figure 12 shows that to determine distribution 1 of the inherent stresses which are a surface loading on the part, it is necessary to subtract distribution 2 from resultant distribution 3. Distribution 3 is calculated by (7), and distribution 2 can be determined by the coordinates of points $A\left(z=h_{p} ; \sigma=\sigma_{p}\right)$ and
$B\left(z=h_{p} ; \sigma=0\right)$ lying on the straight line $B C$. The equation of the straight line $B C$ is:

$$
\frac{z-h_{p}}{h_{e}-h_{p}}=-\frac{\sigma-\sigma_{p}}{\sigma_{p}} .
$$

Based on (13), one can determine the parameters of distribution 2 of the elastic stresses:

$$
\begin{aligned}
& \sigma_{s}^{e}=\sigma_{p}\left(1+\frac{h_{p}}{h_{e}-h_{p}}\right) \\
& \sigma_{e}^{\prime}=\sigma_{p}\left(1-\frac{z^{\prime}-h_{p}}{h_{e}-h_{p}}\right) .
\end{aligned}
$$

After transformations, one obtains the parameters of distribution 1 of the inherent stresses produced by shot peening:

$$
\begin{aligned}
& \sigma_{s}=\sigma_{r_{s}}-\sigma_{p}\left(1+\frac{h_{p}}{h_{e}-h_{p}}\right) ; \\
& \sigma^{\prime}=\sigma_{r}^{\prime}-\sigma_{p}\left(1-\frac{z^{\prime}-h_{p}}{h_{e}-h_{p}}\right) .
\end{aligned}
$$

Equation (15) is used to determine internal force factors $P$ and $z_{c}$ by formulas (4).

\section{CALCULATION METHODS FOR FORMING CONDITIONS}

Conditions for 'peen forming - sanding - shot peening' are determined in order to ensure the prescribed transversal curvature $\kappa_{x}(\kappa=1 / R)$ of the part. Longitudinal component $\kappa_{y}^{f-s-w}$ of the curvature of the part is compensated when bending simultaneously with the prescribed longitudinal curvature of the part $\kappa_{y}$, i.e.:

$$
\kappa^{b}=\kappa_{y}+\kappa_{y}^{f-s-w} .
$$

Equation (17) describes the dependence of the required bending moment on the prescribed curvature of the neutral layer of the sheet part:

$$
M_{j}=0,083 \kappa_{j} K E H_{k}^{\prime 3} ; j, k=x, y,
$$

where $K$ is coefficient characterizing the difference of the dependence between components of stresses and strains under complex stress-strain conditions and dependence corresponding to the uniaxial state of elastic strains where $K=1$. When bending plates, $K=1.125 ; H^{\prime}$ is the thickness of the transversal cross-section of a plate-type part equivalent to the real one having inner stiffeners (ribs, pockets, etc.) by the moment of inertia; $M$ is the bending moment acting on the part in the direction under study and resulting from internal force factors of the forming process. 
For the chosen area of a part with geometrical parameters (curvature and thickness) averaged with prescribed accuracy, let us determine parameters of the complex 'peen forming sanding' process.

To find peen forming parameters, one has to select a combination of shot velocity and exposure time ensuring formation of the required transversal curvature $\kappa_{x}$ of the area. Increase in shot velocity improves the efficiency of peen forming, but deepens indentations which increase labor intensity of sanding. Thus, the combination of technological parameters for 'peen forming - sanding' should minimize the total exposure time calculated by (18):

$$
t_{f-s}=t_{f}+t_{s}=t_{\Sigma}(V)=\min _{V} t_{f-s},
$$

where $t_{f}$ is peen forming time calculated on the basis of the value of transversal curvature $\kappa_{x}$ of the chosen area; $t_{s}$ is sanding time (see (19)) required for the removal of stock $a=h_{i n}^{f}$ from the surface area equal to the peen forming area:

$$
t_{s}=\frac{L_{f}}{s_{s}} \frac{B_{f}}{B_{s}} \frac{a}{a_{0}},
$$

where $L_{f}, B_{f}$ are length and width of the area of peen forming; $s_{s}$ is feed of sanding; $B_{s}$ is width of the area sanded; $a_{0}$ is stock removed when sanding during one work travel.

The dependence of sample arc height $f$ on the peen forming time is calculated by means of the exponential approximation of the saturation curve (20) [8]:

$$
f=a\left[1-\exp \left(-b t^{c}\right)\right],
$$

where $a, b, c$ are approximation parameters calculated on the basis of experimental data.

It is reasonable to assume that elongation of the treated sample will have a similar law of variation and can be approximated by the similar dependence. Thus, variables $f$ and $\varepsilon$ of (4) can be determined by (21):

$$
\begin{aligned}
& \varepsilon=a_{\varepsilon}\left[1-\exp \left(-b_{\varepsilon} t^{c_{\varepsilon}}\right)\right] ; \\
& f=a_{f}\left[1-\exp \left(-b_{f} t^{c_{f}}\right)\right] .
\end{aligned}
$$

To calculate coefficients $a_{f}, b_{f}, c_{f} ; a_{\varepsilon}, b_{\varepsilon}, c_{\varepsilon}$ for peen forming, samples are treated at different values of shot velocity. The process is subdivided into several equal working travels. Each travel is followed by measurements of sample arc height $f$ and elongation $\Delta L$. Coefficients $a_{f}, b_{f}, c_{f} ; a_{\varepsilon}, b_{\varepsilon}, c_{s}$ are calculated using special software by combining experimental and empirical curves with prescribed accuracy. Coefficients corresponding to working values of shot velocity are stored in the database created for each type of the equipment.
Equation (18) is solved by a method of coefficient $a_{f}, b_{f}, c_{f} ; a_{\varepsilon}, b_{\varepsilon}, c_{\varepsilon}$ exhaustion in (21) corresponding to the operating values of shot velocity. The results of solution are values of shot velocity and peen forming time.

Based on the determined modes of peen forming and sanding, one can determine operating conditions and corresponding internal force factors of the processes.

Shot peening parameters are calculated for the chosen areas with widths equal to the width of the part. The treatment conditions for the outer side of the part are prescribed based on the technological recommendations.

Bending moment $M_{e x}^{i n}$ acting on the outer side of the chosen area of the part can be determined by (22):

$$
M_{e j}^{i n}=\frac{1}{B L} \sum_{i=1}^{N_{f}} b_{i}^{f} l_{i}^{f} M_{f-s-e j i}^{i n}, j=x, y,
$$

where $\boldsymbol{B}$ is width of the chosen area; $l^{f}, b^{f}, N_{f}$ are length, width and number of strips treated; $M_{f-s-e}^{\text {in }}$ is bending moment occurring under the effect of internal force factors of 'peen forming - sanding - shot peening' sequence.

Internal components of bending moment $M_{j e}^{\text {out }}, j=x, y$ are calculated by (23):

$$
\begin{aligned}
& M_{e x}^{\text {out }}=\frac{1}{L} \sum_{k=1}^{N_{d}} l_{k}\left(z_{0 x}-h_{k}+z_{c e}^{\text {out }}\right) P_{e}^{\text {out }} ; \\
& M_{e y}^{\text {out }}=\frac{1}{B} \sum_{k=1}^{N_{d}} b_{k}\left(z_{0 y}-h_{k}+z_{c e}^{\text {out }}\right) P_{e}^{\text {out }} .
\end{aligned}
$$

Here, $L, B$ are length and width of the chosen area of the part; $l_{k}$, $b_{k} h_{k}$ are length, width and thickness of the $i$-th element of the chosen area; $N_{d}$ is the number of structural elements of the chosen area; $z_{0 x}, z_{0 y}$ are coordinates of the center of gravity of the chosen area calculated as average values of the coordinates of its cross sections in corresponding directions; $P_{e}^{\text {out }}$ is the internal force acting inside of the chosen area; $z_{c e}^{i n}$ is the distance from the surface to the point of force application $P_{e}^{i n}$.

When shot peen forming, to save the transversal curvature of the part, the bending moment should be (24):

$$
M_{e x}^{i n}=M_{e x}^{\text {out }} .
$$

As a result, one can find internal force $P_{e}^{\text {out }}$ and corresponding conditions for the internal side of the part.

Thus, the values of internal force factors allow one to determine the components for forming a part and find required longitudinal curvature $\boldsymbol{\kappa}^{b}$ using (16) compensating for undesirable strains. 


\section{AUTOMATION OF PEEN FORMING PARAMETERS CALCULATION}

Figure 13 shows a dialogue box of the software module created for automated calculation of peen forming parameters.

The module works as follows [9]. The surface of the electronic module of the part created in Siemens Unigraphics $\mathrm{NX}$ is striped online based on the experience of forming of similar parts (Fig. 14).

In automatic mode, strips are divided into rectangular areas, and the radius of curvature and thickness of the areas are calculated (Fig. 15). The results are the basis for calculation of the bending moment for forming. Conditions are selected in the above mentioned sequence.

Based on the calculations, control software for preliminary forming of the part is created to obtain $70 \ldots 90 \%$ of the prescribed curvature. Based on their experience, the authors can argue that finishing is not a difficult task for an operator and does not need high skills. It allows obtaining any shape accuracy for the part.

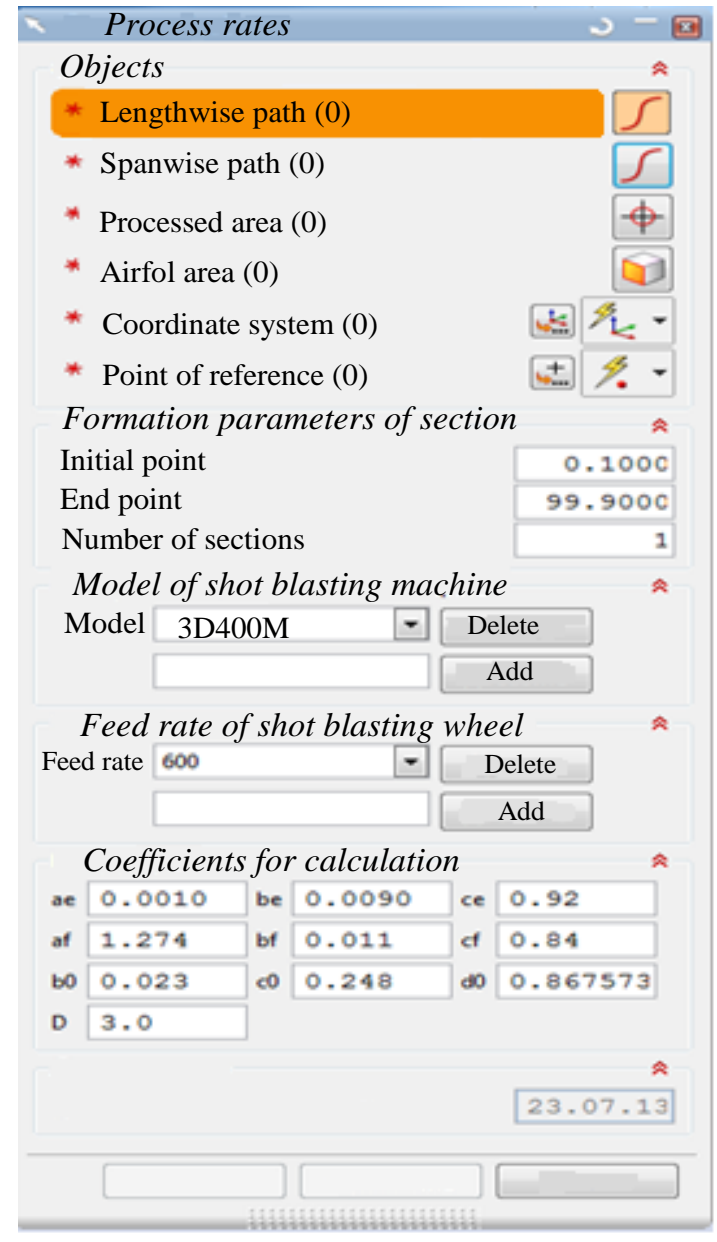

Fig. 13. Dialogue box of the software module for calculation of peenforming parameters

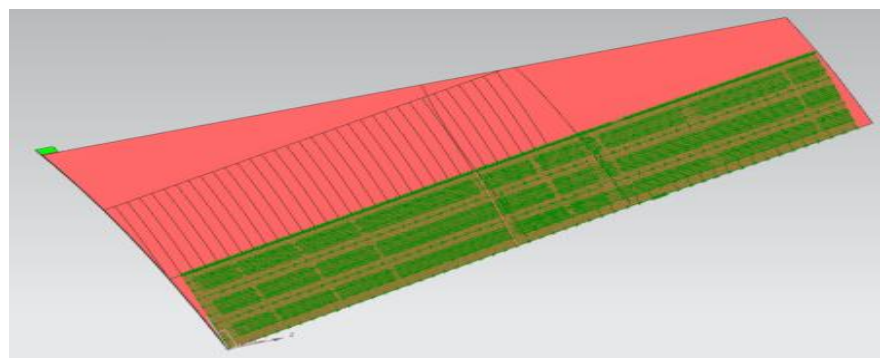

Fig. 14. Sample strips on the skin treated

Peen forming processes can be completely automated after preliminary forming, by intermediate control of shapes, subsequent digitalization of their results and creation of control software for finishing processes.

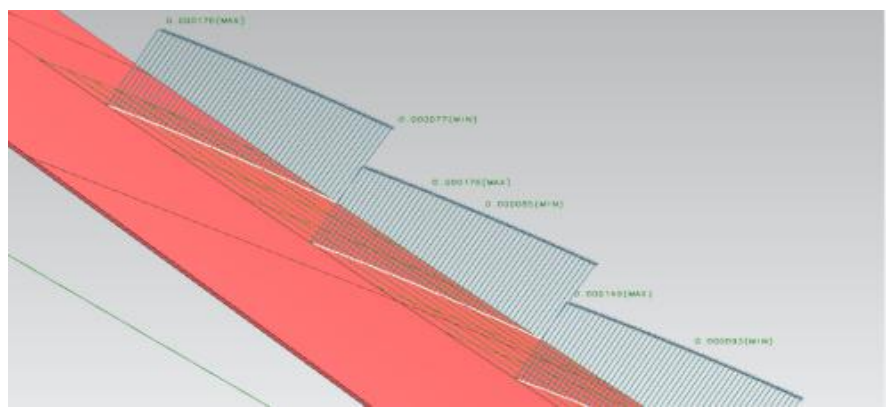

Fig. 15. Calculation of cross section geometric parameters for the skin worked

Thus, one more advantage of the developed method is a combination of automated preliminary forming, manual shape finishing and sanding. It saves time for transportation and setting of large-sized and slender parts which are also difficult technical tasks in batch manufacturing.

\section{References}

[1]. S. Ramati, G. Levasseur, S. Kennerknecht, "Single piece wing skin utilization via advanced peen forming technology", 7-th Int. Conf. on Shot Peening,. Warsaw, Poland, 28 - 30 Sep,. 2000 (ICSP-7), pp. 1-17.

[2]. US Patent №4329862 Int. cl. B24J 5/00; C21D 7/0 Shot Peen Forming of Compound Contours - 18.05.1982

[3]. J.A. Vaccari, "Peen forming enters computer age", American Machinist, 1985, June, pp. 91-94

[4]. Y.I. Zamashchikov, "Machining residual stresses and part distortions", Int. J. Machining and Machinability of Materials, vol. 2 (3), pp. 378 412, 2007.

[5]. V.V. Moskvitin, Plasticity under alternating loadings. Moscow: MGU, 1965. [Plastichnost pri peremenyh nagruzheniyah].

[6]. R. Kopp, K.-P. Hornauer, H.-W. Ball, "Shot-peen-forming, recent technological and theoretical developments", Adv. Surface Treat. Technol. Appl., Eff. Oxford e. a., vol. 2, pp. 21-23, 1986.

[7]. A.E. Pashkov, Technological relations in production of long-length sheet parts. Irkutsk: IrGTU, 2005.

[8]. D. Kirk, "Saturation Curve Analysis and Quality Control", The Shot Peener, Summer, 2006, pp. 24-30.

[9]. Y.S. Andryashina, "Automated calculation of peen shaping technological parameters of large-size panels", Proceedings of Samara Scientific Center, vol. 15, 6(2), pp. 305-308. 